\title{
The group of diffeomorphisms of a non-compact manifold is not regular
}

https://doi.org/10.1515/dema-2018-0001

Received May 24, 2017; accepted December 21, 2017

Abstract: We show that a group of diffeomorphisms $\mathcal{D}$ on the open unit interval $I$, equipped with the topology of uniform convergence on any compact set of the derivatives at any order, is non-regular: the exponential map is not defined for some path of the Lie algebra. This result extends to the group of diffeomorphisms of finite dimensional, non-compact manifold $M$.

Keywords: diffeology, diffeomorphisms, infinite dimensional Lie groups, exponential map

MSC: 22E65, 22E66

\section{Introduction}

In the theory of compact Lie groups, the exponential map defines a chart around the neutral element. This map plays a central role in many aspects of the theory. In infinite dimensional Lie groups, the existence of the exponential map is not straightforward, mostly because of the lack of compact neighbourhood of the neutral element. For this reason, when dealing with infinite dimensional Lie groups, we often work only with regular Lie groups. These are Lie groups that have an exponential map, i.e. a map that integrates any smooth path $v$ on the Lie algebra to a smooth path $g$ on the Lie group via the equation on logarithmic derivatives

$$
d g \cdot g^{-1}=v .
$$

In easy examples, such as Banach Lie groups, the exponential map also defines a chart around the neutral element, but in some other examples such as groups of diffeomorphisms on a compact manifold, it is not the case. This last pathology generates technical difficulties [1, 2].

The notion of a regular Lie group was first described by Omori, motivated by the discovery of nonenlargeable Lie algebras of vector fields [2] after a series of works on the ILH structures of the group of diffeomorphisms of a compact manifold (see e.g. [1]). The terminology of "regular Lie group" was introduced with a second class of examples: groups of Fourier-integral operators in series of articles. For an organized exposition, see [3]. Since these founding examples, despite many efforts, there is still no known example of non-regular Fréchet Lie group. As quoted in [4], there exists many candidates, but there are still significant difficulties in showing that a differential equation on an infinite dimensional Lie group has no solution.

In addition, we have to mention that the universal setting for infinite dimensional geometry seems not to exist. Historically, various authors developed more and more general settings for infinite dimensional "manifolds": Hilbert, Banach, then Fréchet and locally convex manifolds (equipped with atlas), and then raised the necessity to deal with "manifolds" without charts. This phenomenon was even so embarrassing that the precise setting for differential calculus in an infinite dimensional setting has been skipped by several authors for applications, see e.g. [5]. Several definitions and settings have been given by various authors, and the choice

^Corresponding Author: Jean-Pierre Magnot: LAREMA - UMR CNRS 6093, Université d’Angers, 2 Boulevard Lavoisier - 49045 Angers cedex 01 and Lycée Jeanne d’Arc, Avenue de grande bretagne, F-63000 Clermont-Ferrand, France,

E-mail: jean-pierr.magnot@ac-clermont.fr 
that we make is to use diffeological spaces as a maximal category, and Frölicher spaces as an intermediate category for differential geometry $[6,7]$. This choice is still quite controversial but this setting is developed enough for many applications. Moreover, historically, diffeological spaces have been developed by Souriau in the 80 's with the motivation to deal with the precise objects of interest here, that is groups of diffeomorphisms on non-compact, locally compact, smooth manifolds without boundary.

In this short communication, we describe an example of non-regular group of diffeomorphisms $\mathcal{D}$ on the unit interval, which is in the setting of Frölicher Lie groups. The difference is that, in the Frölicher setting, existence of charts is not assumed. For basics on this setting, due to Frölicher and Kriegl, see [4, 8] and e.g. $[6,7,9]$ for a short exposition on Frölicher spaces and Frölicher Lie groups.

This example, which can appear as a toy example, acts as a preliminary result in order to show that the (full) group of diffeomorphisms $\operatorname{Diff}(M)$ is non-regular. Here, $M$ i sa non-compact, finite dimensional manifold $M$, equipped with the topology of uniform convergence of any partial derivative on any compact subset of $M$, called the smooth compact-open topology (or weak topology in [10]). This topology appears as quite natural, and quite minimal compared to the (much stronger) topologies described in [4, section 43.1] (similar to the classical $C^{\infty}$-Whitney topology) and in the more diversified [11], which furnish examples of regular Lie groups (with atlas). In our example, no chart can be actually successfully constructed by the lack of adequate implicit functions theorem in this topology, and also because this example seems not locally homeomorphic to any open subset of a function space for the considered (smooth compact-open) topology. Of course, these considerations are still open questions. Indeed, at present it is unknown whether one can get these local homeomorphism properties for $\mathcal{D}$.

Our method of proof is inspired by:

- $\quad$ first the example of non-integrable Lie subalgebra of $\operatorname{Vect}\left(S^{1}\right)$ due to Omori [2], where non-integrability is due to the existence of translations,

- secondly the non-existence of horizontal lifts for connections on fiber bundles when the typical fiber is not compact, see [12]. Moreover, if the typical fiber is compact, connections on the considered fiber bundle are in one-to-one correspondence with connections on a principal bundle with structure group a (regular) group of diffeomorphisms, see e.g. [4].

We need to mention that the example that we develop seems already known in the mathematical literature,but nowhere stated clearly to our knowledge so that we feel the need of a rigorous description of the announced phenomenon: a constant path on the Lie algebra is not integrable into a path of the group. This is done in three steps:

- first summarize the settings that have been developed to enable a rigorous differential geometry on groups of diffeomorphisms on non-compact manifolds (section 1), namely diffeological spaces and Frölicher spaces, applied to our example of group of diffeomorphisms on the open unit interval;

- secondly show that the constant map on the unit interval is in $T_{I d_{00 ; 1}} \mathcal{D}$, and that this element understood as a constant path cannot integrate in $\mathcal{D}$ by an argument of "translation semi-group" discovered by [2] for a different goal;

- finally embedding $\mathcal{D}$ into $\operatorname{Diff}(M)$, for a non-compact manifold $M$, in a way such that the translation semi-group required on $\mathcal{D}$ cannot be described as a semi-group of transformations on $M$, we get a direct proof, new to our knowledge, of the following result:

Theorem 0.1. Let $M$ be a locally compact, non-compact manifold. Then the group Diff(M), equipped with its functional diffeology, is a diffeological Lie group which is non-regular. 


\section{Preliminaries}

\subsection{Souriau's diffeological spaces and Frölicher spaces}

Definition 1.1. [13-15] Let $X$ be a set.

- A p-parametrization of dimension $p$ (or $p$-plot) on $X$ is a map from an open subset $O$ of $\mathbb{R}^{p}$ to $X$.

- A diffeology on $X$ is a set $\mathcal{P}$ of parametrizations on $X$, called plots of the diffeology, such that, for all $p \in \mathbb{N}$, - any constant map $\mathbb{R}^{p} \rightarrow X$ is in $\mathcal{P}$;

- Let $I$ be an arbitrary set of indexes, let $\left\{f_{i}: O_{i} \rightarrow X\right\}_{i \in I}$ be a family of compatible maps that extend to a $\operatorname{map} f: \bigcup_{i \in I} O_{i} \rightarrow X$. If $\left\{f_{i}: O_{i} \rightarrow X\right\}_{i \in I} \subset \mathcal{P}$, then $f \in \mathcal{P}$.

- Let $f \in \mathcal{P}$ defined on $O \subset \mathbb{R}^{p}$. Let $q \in \mathbb{N}, O^{\prime}$ to be an open subset of $\mathbb{R}^{q}$, and $g$ to be a smooth map (in the usual sense) from $O^{\prime}$ to $O$. Then $f \circ g \in \mathcal{P}$.

- If $\mathcal{P}$ is a diffeology on $X$, then $(X, \mathcal{P})$ is called a diffeological space.

Let $(X, \mathcal{P})$ and $\left(X^{\prime}, \mathcal{P}^{\prime}\right)$ be two diffeological spaces; a map $f: X \rightarrow X^{\prime}$ is differentiable (=smooth) if and only if $f \circ \mathcal{P} \subset \mathcal{P}^{\prime}$.

Remark 1.2. Any diffeological space $(X, \mathcal{P})$ can be endowed with the weakest topology such that all the maps that belong to $\mathcal{P}$ are continuous. This topology is called the D-topology, see [16].

We now introduce Frölicher spaces, see [8], using the terminology defined in [4].

Definition 1.3. - A Frölicher space is a triple $(X, \mathcal{F}, \mathcal{C})$ such that : - $\mathcal{C}$ is a set of paths $\mathbb{R} \rightarrow X$; - $\mathcal{F}$ is the set of functions from $X$ to $\mathbb{R}$, such that the function $f: X \rightarrow \mathbb{R}$ is in $\mathcal{F}$ if and only if for any $c \in \mathcal{C}, f \circ c \in C^{\infty}(\mathbb{R}, \mathbb{R})$;

- A path $c: \mathbb{R} \rightarrow X$ is in $\mathcal{C}$ (i.e. is a contour) if and only if for any $f \in \mathcal{F}, f \circ c \in C^{\infty}(\mathbb{R}, \mathbb{R})$.

- Let $(X, \mathcal{F}, \mathcal{C})$ and $\left(X^{\prime}, \mathcal{F}^{\prime}, \mathcal{C}^{\prime}\right)$ be two Frölicher spaces; a map $f: X \rightarrow X^{\prime}$ is differentiable (=smooth) if and only if $\mathcal{F}^{\prime} \circ f \circ \mathcal{C} \subset C^{\infty}(\mathbb{R}, \mathbb{R})$.

Any family of maps $\mathcal{F}_{g}$ from $X$ to $\mathbb{R}$ generates a Frölicher structure $(X, \mathcal{F}, \mathcal{C})$ by setting, after [4]:

- $\mathcal{C}=\left\{c: \mathbb{R} \rightarrow X\right.$ such that $\left.\mathcal{F}_{g} \circ c \subset C^{\infty}(\mathbb{R}, \mathbb{R}) ;\right\}$

- $\mathcal{F}=\left\{f: X \rightarrow \mathbb{R}\right.$ such that $\left.f \circ \mathcal{C} \subset C^{\infty}(\mathbb{R}, \mathbb{R})\right\}$.

In this case we call $\mathcal{F}_{g}$ a generating set of functions for the Frölicher structure $(X, \mathcal{F}, \mathcal{C})$. One easily see that $\mathcal{F}_{g} \subset \mathcal{F}$. This notion will be useful in the sequel to describe in a simple way a Frölicher structure. A Frölicher space $(X, \mathcal{F}, \mathcal{C})$ carries a natural topology, which is the pull-back topology of $\mathbb{R}$ via $\mathcal{F}$. We note that in the case of a finite dimensional differentiable manifold $X$ we can take $\mathcal{F}$ the set of all smooth maps from $X$ to $\mathbb{R}$, and $\mathcal{C}$ the set of all smooth paths from $\mathbb{R}$ to $X$. In this case the underlying topology of the Frölicher structure is the same as the manifold topology [4]. In the infinite dimensional case, there is to our knowledge no complete study of the relation between the Frölicher topology and the manifold topology; our intuition is that these two topologies can differ.

We also remark that if $(X, \mathcal{F}, \mathcal{C})$ is a Frölicher space, we can define a natural diffeology on $X$ by using the following family of maps $f$ defined on open domains $D(f)$ of Euclidean spaces (see [6]):

$$
\mathcal{P}_{\infty}(\mathcal{F})=\coprod_{p \in \mathbb{N}}\left\{f: D(f) \rightarrow X ; \mathcal{F} \circ f \in C^{\infty}(D(f), \mathbb{R}) \quad \text { (in the usual sense) }\right\} .
$$

If $X$ is a differentiable manifold, this diffeology has been called the nébuleuse diffeology by J.-M. Souriau [13] or nebulae diffeology in [14]. We can easily show the following:

Proposition 1.4. [6] Let $(X, \mathcal{F}, \mathcal{C})$ and $\left(X^{\prime}, \mathcal{F}^{\prime}, \mathrm{C}^{\prime}\right)$ be two Frölicher spaces. A map $f: X \rightarrow X^{\prime}$ is smooth in the sense of Frölicher if and only if it is smooth for the underlying diffeologies $\mathcal{P}_{\infty}(\mathcal{F})$ and $\mathcal{P}_{\infty}\left(\mathcal{F}^{\prime}\right)$. 
Thus, we can also state:

$\overline{\text { smooth manifold } \Rightarrow \text { Frölicher space } \Rightarrow \text { diffeological space }}$

A deeper analysis of these implications has been given in [7]. The next remark is inspired by this work and by [6]; it is based on [4, p.26, Boman's theorem].

Remark 1.5. We notice that the set of contours $\mathcal{C}$ of the Frölicher space $(X, \mathcal{F}, \mathcal{C})$ does not give us a diffeology, because a diffelogy needs to be stable under restriction of domains. In the case of paths in $\mathcal{C}$ the domain is always $\mathbb{R}$ where as the domain of 1-plots can (and has to) be any interval of $\mathbb{R}$. However, $\mathcal{C}$ defines a "minimal diffeology" $\mathcal{P}_{1}(\mathcal{F})$ whose plots are smooth parameterizations which are locally of the type $c \circ g$, where $g \in$ $\mathcal{P}_{\infty}(\mathbb{R})$ and $c \in \mathcal{C}$. Within this setting, we can replace $\mathcal{P}_{\infty}$ by $\mathcal{P}_{1}$ in Proposition 1.4.

We also remark that given an algebraic structure, we can define a corresponding compatible diffeological structure. For example following [14, pp. 66-68], an $\mathbb{R}$-vector space equipped with a diffeology is called a diffeological vector space if addition and scalar multiplication are smooth. An analogous definition holds for Frölicher vector spaces.

Remark 1.6. Frölicher and Gateaux smoothness are the same notion if we restrict to a Fréchet context. Indeed, for a smooth map $f:\left(F, \mathcal{P}_{1}(F)\right) \rightarrow \mathbb{R}$ defined on a Fréchet space with its 1-dimensional diffeology, we have that $\forall(x, h) \in F^{2}$, the map $t \mapsto f(x+t h)$ is smooth as a classical map in $\mathcal{C}^{\infty}(\mathbb{R}, \mathbb{R})$. By the way, it is Gateaux smooth. The converse is obvious.

Diffeologies on cartesian products, projective limits, quotients, subsets as well as pull-back and push-forward diffeologies are described in [14, Chapter 1$]$. The reader can refer also to $[13]$ or $[9,17]$ for faster exposition.

\subsection{Functional diffeology}

Let $(X, \mathcal{P})$ and $\left(X^{\prime}, \mathcal{P}^{\prime}\right)$ be two diffeological spaces. Let $M \subset C^{\infty}\left(X, X^{\prime}\right)$ be a set of smooth maps. The functional diffeology on $S$ is the diffeology $\mathcal{P}_{S}$ made of plots

$$
\rho: D(\rho) \subset \mathbb{R}^{k} \rightarrow S
$$

such that, for each $p \in \mathcal{P}$, the maps $\Phi_{\rho, p}:(x, y) \in D(p) \times D(\rho) \mapsto \rho(y)(x) \in X^{\prime}$ are plots of $\mathcal{P}^{\prime}$. With this definition, we have the classical fundamental propertiy for calculus of variations:

Proposition 1.7. [14] Let $X, Y, Z$ be diffeological spaces,

$$
C^{\infty}(X \times Y, Z)=C^{\infty}\left(X, C^{\infty}(Y, Z)\right)=C^{\infty}\left(Y, C^{\infty}(X, Z)\right)
$$

as diffeological spaces equipped with functional diffeologies.

\subsection{Tangent space}

There are actually two main definitions, (2) and (3) below, of the tangent space of a diffeological space:

1. The internal tangent cone defined in [18]. For each $x \in X$, we consider

$$
C_{x}=\left\{c \in C^{\infty}(\mathbb{R}, X) \mid c(0)=x\right\}
$$

and take the equivalence relation $\mathcal{R}$ given by

$$
c \mathcal{R} c^{\prime} \Leftrightarrow \forall f \in C^{\infty}(X, \mathbb{R}),\left.\partial_{t}(f \circ c)\right|_{t=0}=\left.\partial_{t}\left(f \circ c^{\prime}\right)\right|_{t=0} .
$$


The internal tangent cone at $x$ is the quotient

$$
{ }^{i} T_{X} X=C_{X} / \mathcal{R} .
$$

If $V=\left.\partial_{t} c(t)\right|_{t=0} \in{ }^{i} T_{X} X$, we define the simplified notation

$$
D f(V)=\left.\partial_{t}(f \circ c)\right|_{t=0} .
$$

2. The internal tangent space at $x \in X$ described in [19].

3. The external tangent space ${ }^{e} T X$, defined as the set of derivations on $C^{\infty}(X, \mathbb{R})[4,14]$.

It is shown in [18] that the internal tangent cone at a point $x$ is not a vector space in many examples. This motivates [19]. For finite dimensional manifold, definitions (1), (2) and (3) coincide. For more comparisons, see [4, section 28] for a comparison for infinite dimensional manifolds and also [19].

\subsection{Regular Lie groups}

Definition 1.8. Let $G$ be a group, equiped with a diffeology $\mathcal{P}$. We call it diffeological group if both multiplication and inversion are smooth.

The same definitions hold for Frölicher groups. Let us now recall [20, Proposition 1.6.], which shows that the distinction between internal tangent cone and internal tangent space is not necessary for diffeological groups.

Proposition 1.9. Let $G$ be a diffeological group. Then the tangent cone at the neutral element $T_{e} G$ is a diffeological vector space.

Following Iglesias-Zemmour [14] who does not assert that arbitrary diffeological groups have a Lie algebra, we restrict ourselves to a smaller class of diffeological groups which have such a tangent space at the neutral element. Intuitively speaking, the diffeological group $G$ is a diffeological Lie group if and only if the derivative of the Adjoint action of $G$ on ${ }^{i} T_{e} G$ defines a Lie bracket. In this case, we call ${ }^{i} T_{e} G$ the Lie algebra of $G$, that we note generically by $\mathfrak{g}$. One crucial question consists in giving a technical condition which ensures the classical properties of Adjoint and adjoint actions, e.g.:

- Let $(X, Y) \in \mathfrak{g}^{2}, X+Y=\partial_{t}(c . d)(0)$ where $c, d \in \mathcal{C}^{2}, c(0)=d(0)=e_{G}, X=\partial_{t} c(0)$ and $Y=\partial_{t} d(0)$.

- Let $(X, g) \in \mathfrak{g} \times G, A d_{g}(X)=\partial_{t}\left(g c g^{-1}\right)(0)$ where $c \in \mathcal{C}, c(0)=e_{G}$, and $X=\partial_{t} c(0)$.

- Let $(X, Y) \in \mathfrak{g}^{2},[X, Y]=\partial_{t}\left(A d_{c(t)} Y\right)$ where $c \in \mathcal{C}, c(0)=e_{G}, X=\partial_{t} c(0)$.

According to [9] (which deals with Frölicher Lie groups), one can assume only that the desired properties are fulfilled, leaving technicities for specific examples. One criteria has been given in [20, Definition 1.13 and Theorem 1.14] but this is not necessary here since in the framework that we consider, the properties of the Lie bracket will arise naturally and directly. For these reasons, we give the following definition:

Definition 1.10. The diffeological group $G$ is a diffeological Lie group if and only if the derivative of the Adjoint action of $G$ on ${ }^{i} T_{e} G$ defines a smooth Lie bracket. In this case, we call ${ }^{i} T_{e} G$ the Lie algebra of $G$, that we note generically $\mathfrak{g}$.

Let us now concentrate on diffeological Lie groups, and in this case we note $\mathfrak{g}={ }^{i} T_{e} G$. The basic properties of adjoint, coadjoint actions, and of Lie brackets, remain globally the same as in the case of finite-dimensional Lie groups, and the proofs are similar. We only need to replace charts by plots of the underlying diffeologies (see e.g. [20] for further details, and [?] for the case of Frölicher Lie groups), as soon as one has checked that the Lie algebra $\mathfrak{g}$ is a diffeological Lie algebra, i.e. a diffeological vector space with smooth Lie bracket.

Definition 1.11. [20] A diffeological Lie group $G$ with Lie algebra $\mathfrak{g}$ is called regular if and only if there is a smooth map

$$
\operatorname{Exp}: C^{\infty}([0 ; 1], \mathfrak{g}) \rightarrow C^{\infty}([0,1], G)
$$


such that $g(t)=\operatorname{Exp}(v(t))$ is the unique solution of the differential equation

$$
\left\{\begin{array}{l}
g(0)=e, \\
\frac{d g(t)}{d t} g(t)^{-1}=v(t)
\end{array}\right.
$$

We define the exponential function as follows:

$$
\begin{aligned}
\operatorname{Exp}: \mathfrak{g} & \rightarrow G \\
v & \mapsto \exp (v)=g(1),
\end{aligned}
$$

where $g$ is the image by Exp of the constant path $v$.

\subsection{Groups of diffeomorphisms}

Let $M$ be a locally compact, non-compact manifold, which is assumed to be Riemannian without restriction, equipped with its nébuleuse diffeology. We equip the group of diffeomorphisms Diff( $M$ ) with the topology of convergence of the derivatives an any order, uniformly on each compact subset of $M$, usually called $C^{\infty}$-compact-open topology or weak topology in [10]. Traditionnally, $\operatorname{Vect}(M)$ is given as the Lie algebra of $\operatorname{Diff}(M)$, but [4, section 43.1] shows that this strongly depends on the topology of $\operatorname{Diff}(M)$. Indeed, the Lie algebra of vector fields described in [4, section 43.1] is the Lie algebra of compactly supported vector fields, which is not the (full) Lie algebra $\operatorname{Vect}(M)$. In another context, when $M$ is compact, $\operatorname{Vect}(M)$ is the Lie algebra of $\operatorname{Diff}(M)$, which can be obtained by Omori's regularity theorems [1,3] and recovered in [19]. What is well known is that infinitesimal actions (i.e. elements of the internal tangent space at identity) of Diff( $M$ ) on $C^{\infty}(M, \mathbb{R})$ generates vector fields, viewed as order 1 differential operators. The bracket on vector fields is given by

$$
(X, Y) \in \operatorname{Vect}(M) \mapsto[X, Y]=\nabla_{X} Y-\nabla_{Y} X,
$$

where $\nabla$ is the Levi-Civita connection on TM. This is a Lie bracket, stable under the Adjoint action of Diff(M). Moreover, the compact-open topology on $\operatorname{Diff}(M)$ generates a corresponding $C^{\infty}$-compact-open topology on $\operatorname{Vect}(M)$. This topology is itself the $D$-topology for the the functional diffeology on $\operatorname{Diff}(M)$. Following [20, Definition 1.13 and Theorem 1.14], $\operatorname{Vect}(M)$ equipped with the $C^{\infty}$ compact-open topology is a Fréchet vector space, and the Lie bracket is smooth. Moreover, we feel the need to remark that the evaluation maps

$$
T^{\star} M \times \operatorname{Vect}(M) \rightarrow \mathbb{R}
$$

separate $\operatorname{Vect}(M)$. Thus $\operatorname{Diff}(M)$ is a diffeological Lie group matching with the criteria of [20, Definition 1.13 and Theorem 1.14], and for the functional diffeology, with Lie algebra $\mathfrak{g} \subset \operatorname{Vect}(M)$.

\section{A non-regular group of diffeomorphims of the unit interval}

\subsection{Premilinaries}

Let $F$ be the vector space of smooth maps $f \in C^{\infty}(] 0 ; 1[; \mathbb{R})$. We equip $F$ with the following semi-norms:

For each $(n, k) \in \mathbb{N}^{*} \times \mathbb{N}$,

$$
\|f\|_{n, k}=\sup _{\frac{1}{n+1} \leq x \leq \frac{n}{n+1}}\left|D_{x}^{k} f\right| .
$$

This is a Fréchet space, and its topology is the smooth compact-open topology, which is the $D$-topology of the compact-open diffeology. Let

$$
\mathcal{A}=\left\{f \in C^{\infty}(] 0 ; 1[;] 0 ; 1[) \mid \lim _{x \rightarrow 1} f(x)=1 \wedge \lim _{x \rightarrow 0} f(x)=0\right\} .
$$


Finally, we set

$$
\mathcal{D}=\left\{f \in \mathcal{A} \mid \inf _{x \in] 0 ; 1[} f^{\prime}(x)>0\right\} .
$$

$\mathcal{D}$ is a contractible set of diffeomorphisms of the open interval ]0; 1 [ which is an (algebraic) group for composition of functions. Composition of maps, and inversion, is smooth for the functional diffeology. Unfortunately, $\mathcal{D}$ is not open in $\mathcal{A}$. As a consequence, we are unable to prove that it is a Fréchet Lie group. However, considering the smooth diffeology induced on $\mathcal{D}$ by $\mathcal{A}$, the inversion is smooth. As a consequence, $\mathcal{D}$ is (only) a Frölicher Lie group.

\subsection{A non-integrable path of the Lie algebra}

Let us consider the standard mollifier

$$
\phi(u)=\frac{1}{K} e^{\frac{1}{u^{2}-1}}
$$

defined for $u \in]-1 ; 1[$, with

$$
K=\int_{-1}^{1} e^{\frac{1}{u^{2}-1}} d u>0,4
$$

and extended smoothly to $\mathbb{R}$ by setting $\phi(u)=0$ whenever $u \notin]-1 ; 1\left[\right.$. We set $\phi_{\alpha}(u)=\frac{1}{\alpha} \phi\left(\frac{x}{\alpha}\right)$. Let us define, with 1 the standard characteristic function of a set,

$$
\begin{aligned}
c_{t}(x)= & x+t\left(\mathbf{1}_{[|t| ; 1-|t|]}(x) \star \phi_{|t|}(x)\right) \\
= & \left\{\begin{array}{cl}
\forall x<2|t|, & c_{t}=x+t \int_{|t|-x}^{|t|} \phi_{|t|}(u) d u, \\
\forall x \in[2|t| ; 1-2|t|], & c_{t}(x)=x+t, \\
\forall x>1-2|t|, & c_{t}=x+t \int_{-|t|}^{1-|t|-x} \phi_{|t|}(u) d u,
\end{array}\right.
\end{aligned}
$$

where ${ }^{\star}$ is the standard convolution in the $x$-variable, and $-1 / 4<t<1 / 4$. For $t \neq 0,(t, x) \mapsto c_{t}(x)$ is smooth. Moreover, since

$$
\sup \phi<\frac{e^{-1}}{0,4}<1,
$$

for fixed $t$, one can easily check that $\partial_{x} c_{t}>0$, which shows that $\left.\forall t \in\right]-1 / 4 ; 1 / 4\left[, c_{t} \in \mathcal{D}\right.$.

Theorem 2.1. The path $t \mapsto c_{t}$ is of class $C^{\infty}(]-1 / 4 ; 1 / 4[, \mathcal{D})$. Moreover,

$$
\left.\partial_{t} c_{t}\right|_{t=0}=\mathbf{1}_{0 ; 1}[
$$

is a constant map.

Proof. Let $x \in] 0 ; 1\left[\right.$. Let us now check smoothness of $(t, x) \mapsto c_{t}(x)$ at $(0 ; x)$. Let $\alpha=\min \{x, 1-x\}$ and let us restrict our study on the open subset $]-\frac{\alpha}{4} ; \frac{\alpha}{4}[\times] x-\frac{\alpha}{2} ; x+\frac{\alpha}{2}[$. For $t \in]-\frac{\alpha}{4} ; \frac{\alpha}{4}[$, we have that

$$
c_{t}(u)=x+t
$$

whenever

$$
u \in] x-\frac{\alpha}{2} ; x+\frac{\alpha}{2}[\subset] \frac{\alpha}{2} ; 1-\frac{\alpha}{2}[\subset] 2|t| ; 1-2|t|[.
$$

By the way, $(u, t) \mapsto c_{t}(u)$ is smooth on $]-\frac{\alpha}{4} ; \frac{\alpha}{4}[x] x-\frac{\alpha}{2} ; x+\frac{\alpha}{2}[$ and hence it is smooth everywhere. By direct differentiation,

$$
\left.\partial_{t} c_{t}(x)\right|_{t=0}=1
$$


Now, we get the following:

Theorem 2.2. There exists a smooth path $v$ on $T_{I d_{00 ; 1}} \mathcal{D}$ such that no smooth path $g$ on $\mathcal{D}$ satisfies the equation

$$
\partial_{t} g \circ g^{-1}=v .
$$

Proof. Let $v$ be the constant path equal to 1. Let $t \mapsto g_{t}$ be a solution of the last equation and let $\left.x \in\right] 0 ; 1[$. Then we have, $\forall y \in] 0 ; 1\left[\right.$, setting $x=g_{t}(y)$,

$$
\partial_{t} g_{t}(y)=\left(\partial_{t} g_{t}\right) \circ g_{t}^{-1}(x)=1,
$$

and by the way,

$$
\forall(t, y) \in]-1 / 4 ; 1 / 4[\times] 0 ; 1\left[, \partial_{t} c_{t}(y)=1 \text { and } c_{o}(y)=y\right.
$$

so that the only possible solution is the translation $g_{t}(x)=x+t$. We have

$$
\forall t>0, g_{t} \notin \mathcal{A}
$$

so that

$$
\forall t>0, g_{t} \notin \mathcal{D} \text {. }
$$

As a consequence, we get the announced result:

Theorem 2.3. $\mathcal{D}$ is a non-regular Frölicher Lie group.

\subsection{Final remark: $\operatorname{Diff}(M)$ is a non-regular diffeological Lie group}

We now finish by giving a direct proof for the non-regularity of the group of diffeomorphisms of a non-compact boundaryless manifold. Let $M$ be a smooth manifold, for which there is an embedding

$$
e:] 0 ; 1[\rightarrow E
$$

for which $\operatorname{Im}(e)$ is closed in $M$. We also assume that this embedding can be extended to a so-called "thick path", whose image will be a tubular neighbourhood of $\operatorname{Im}(e)$. These are the main conditions that are needed, which are fulfilled when $M$ is $n$-dimensional. Under these conditions, following the "smooth tubular neighbourhood theorem" (see e.g. [21]) we can assume that there is a parametrization of the closed tubular neighbourhood under consideration via an embedding

$$
E:] 0 ; 1\left[\times B_{n-1} \rightarrow M,\right.
$$

where $B_{n-1}$ is the Euclidian $n-1$ dimensional unit ball and $E(x, 0)=e(x)$. We set $\phi: \mathbb{R} \rightarrow \mathbb{R}_{+}$a smooth function with support in $[-1 ; 1]$ and such that $\phi=1$ on a neighbourhood of 0 . We also parametrize $B_{n-1}$ via spherical coordinates $\left(r, \theta_{1}, \ldots \theta_{n-1}\right)$. Under these conditions, we consider the path

$$
C_{t}: \mathbb{R} \rightarrow \operatorname{Diff}(M)
$$

defined by

$$
C_{t}(x)=\left\{\begin{array}{cl}
x & \text { if } x \notin \operatorname{Im}(E), \\
E\left(c_{\phi(r) . t}\left(x^{\prime}\right), r, \theta_{1}, \ldots, \theta_{n-1}\right) & \text { if } x=E\left(x^{\prime}, r, \theta_{1}, \ldots, \theta_{n-1}\right) .
\end{array}\right.
$$

We have that $\left.\partial_{t} C_{t}(x)\right|_{t=0}$ is a smooth vector field, which is equal to $e_{\star}(\mathbf{1})$ when $r=0$. As a consequence, we have

Theorem 2.4. The vector field $\left.\partial_{t} C_{t}(x)\right|_{t=0}$ has no global flow on $M$ and hence Diff $(M)$ is a non-regular Frölicher Lie group. 
Proof. We investigate the flow of $\left.\partial_{t} C_{t}(x)\right|_{t=0}$ on $\operatorname{Im}(e)$ and we have that

$$
e^{\star}\left(\left.\partial_{t} C_{t}(x)\right|_{t=0}\right)=\mathbf{1} \times\{0\} \in e^{\star} T M=C^{\infty}(] 0 ; 1\left[; \mathbb{R} \times \mathbb{R}^{n-1}\right) .
$$

Thus the flow along $\operatorname{Im}(e)$ must be $t \mapsto e(x+t)$. Since $\operatorname{Im}(e)$ is closed in $M$, this flow does not extend to a flow in $M$.

Acknowledgements: the author would like to thank the two anonymous referees, as well as Daniel Christensen, who helped to improve the last version of this paper with very interesting comments and questions.

\section{References}

[1] Omori H., Groups of diffeomorphisms and their subgroups, Trans. Amer. Math. Soc., 1973, 179, 85-122

[2] Omori H., A remark on nonenlargeable Lie algebras, J. Math. Soc. Japan, 1981, 33(4), 707-710

[3] Omori H., Infinite dimensional Lie groups, AMS Translations of Mathematical Monographs, Amer. Math. Soc., Providence, R.I., 1997, 158

[4] Kriegl A., Michor P. W., The convenient setting for global analysis, AMS Math. Surveys and Monographs, AMS, Providence, 1997, 53

[5] Khesin B., Wendt R., Geometry of infinite dimensional groups, Springer, 2008

[6] Magnot J.-P., Difféologie du fibré d'Holonomie en dimension infinie, C. R. Math. Soc. Roy. Can., 2006, 28(4), 121-127

[7] Watts J., Diffeologies, differentiable spaces and symplectic geometry, University of Toronto, PhD thesis, 2013, arXiv:1208.3634v1

[8] Frölicher A., Kriegl A., Linear spaces and differentiation theory, Wiley series in Pure and Applied Mathematics, Wiley Interscience, 1988

[9] Magnot J.-P., Ambrose-Singer theorem on diffeological bundles and complete integrability of the KP equation, Int. J. Geom. Meth. Mod. Phys., 2013, 10(9), DOI: 10.1142/S0219887813500436

[10] Hirsch M., Differential topology, Springer, 1997

[11] Kriegl A., Michor P. W., Rainer A., An exotic zoo of diffeomorphism groups on $\mathbb{R}^{n}$, Ann. Global Anal. Geom., 2015, 47(2), 179-222

[12] Kolar I., Michor P. W., Slovak J., Natural operations in differential geometry, Springer, 1993

[13] Souriau J.-M., Un algorithme générateur de structures quantiques, Astérisque (hors série), 1985, 341-399

[14] Iglesias-Zemmour P., Diffeology, Mathematical Surveys and Monographs, 2013, 185

[15] Neeb K.-H., Towards a Lie theory of locally convex groups, Japanese J. Math., 2006, 1, 291-468

[16] Christensen D., Sinnamon G., Wu E., The D-topology for diffeological spaces, Pacific J. Math., 2014, 272(1), 87-110

[17] Magnot J.-P., q-deformed Lax equations and their differential geometric background, Lambert Academic Publishing, Saarbrucken, Germany, 2015

[18] Dugmore D., Ntumba P., On tangent cones of Frölicher spaces, Quaetiones Mathematicae, 2007, 30(1), 67-83

[19] Christensen D., Wu E., Tangent spaces and tangent bundles for diffeological spaces, Cahiers de Topologie et Géométrie Différentielle, 2016, LVII, 3-50

[20] Leslie J., On a diffeological group realization of certain generalized symmetrizable Kac-Moody Lie algebras, J. Lie Theory, 2003, 13, 427-442

[21] Berger M., A panoramic overview of Riemannian geometry, Springer, 2003 